\title{
Selective affective biasing in recognition memory in the irritable bowel syndrome
}

\author{
J E Gomborone, P A Dewsnap, G W Libby, M J G Farthing
}

\begin{abstract}
The cognitive model of depression assigns a central role to negatively biased information processing in the pathogenesis of the emotional disorder. The relationship between depression and irritable bowel syndrome (IBS) was explored from a cognitive perspective. A word recognition memory task was constructed: subjects had to memorise and subsequently recognise a set of emotionally loaded stimulus words with either positive, neutral, or negative connotations. Four age matched groups participated - 30 IBS patients, 28 depressed patients, 28 patients with organic gastrointestinal disease, and 30 healthy volunteers. The depressed patients, as would be expected, showed a significant bias in favour of emotionally negative words $(p<0.05)$ : the IBS patients showed the same negative bias. In addition the IBS patients made significantly more false-positive type errors in recognising emotionally negative words than either the depressed patients $(p<0.05)$ or the healthy volunteers $(p<0.01)$. This suggests that the IBS patients have a peculiar confirmatory bias for negative material. This may have clinical relevance in terms of the IBS patients' evaluation of their own abdominal sensory experience.

(Gut 1993; 34: 1230-1233)
\end{abstract}

The aetiology of the irritable bowel syndrome (IBS) is unknown but psychological factors have often been implicated. A number of studies using self report symptom questionnaires and diagnostic interviews, developed for use on psychiatric populations, have found high rates of affective symptoms in IBS patients. The best of these studies estimate the prevalence of frank psychiatric disorder to be $40-50 \% .^{12}$

We report a study in which IBS patients were investigated from the perspective of their cognitive psychopathology not their psychiatric symptoms.

The cognitive model of affective disorder proposes that the features of depression can be divided into three categories - the emotional, the behavioural, and the cognitive - and that the latter exert a fundamental influence in initiating, or at least perpetuating, the pathological mood state. $^{3}$ The cognitive features of depression encompass the characteristic ways in which depressed people attend to, perceive, evaluate, and remember information. These are held to be directed by a set of negative schemata that selectively bias information processing so that affected individuals construe themselves, their environment, and their future in a negative light.

While a generally negative outlook is readily discernible in clinical practice with depressed people, other features are more clearly demonstrated under experimental conditions. For example, several studies on memory in people with depression have shown that there is selective affective biasing of recall in favour of material with negative emotional connotations. ${ }^{+}$ This effect applies not only to complex biographical material but even to lists of adjectives. ${ }^{5}$

In this study we investigated recognition memory for lists of adjectives to test the hypothesis that there would be similarities in cognitive psychopathology in patients with IBS and depression. To control for the possible confounding influence of ongoing physical problems we included a comparison group of patients with organic gastrointestinal disease, as well as a group of normal volunteers.

\section{Methods}

SUBJECTS

Altogether 116 subjects were included in the study ( 35 men and 81 women). Thirty patients had a clinical diagnosis of irritable bowel syndrome based on the Manning criteria ${ }^{6}$ (median age 36 years, range 19-64); 28 were depressed and fulfilled DSM-III-R criteria for major depression ${ }^{7}$ (median age 38 years, range 21-64); 28 patients had organic gastrointestinal disease (nine had ulcerative colitis and 19 Crohn's disease (median age 27 years, range 19-54)); and 30 were healthy volunteers (median age 35 years, range 19-60). All patients were randomly selected from outpatient clinic populations and, in the case of the IBS and organic disease patients, had had gastrointestinal symptoms for at least one year. The male:female ratio remained approximately $1: 2$ across all groups.

\section{WORD RECOGNITION MEMORY TEST}

A word recognition memory test was administered. All subjects signed a consent form which described the procedure and explained that the purpose of the study was to investigate attention and memory in different disorders. The test itself consisted of two parts.

In the first part each subject was presented with 24 words on flash cards which they were asked to read out and memorise. These words were of varying emotional connotation (eight neutral, eight positive, eight negative) taken from the Anderson 555 Personality Trait word list. ${ }^{8}$ The list of words that we used is shown in the Table. The words used were controlled for length, meaningfulness, and the frequency with which they occur in the English language. ${ }^{9}$ The same set of words was used for each subject but 
the order in which they were presented was randomised.

The second part, the recognition task, followed 15 minutes later. The subjects were

TABLE Words with different emotional connotations

\begin{tabular}{lll}
\hline Neutral & Positive & Negative \\
\hline Changeable & Dependable & Irritating \\
Cautious & Cheerful & Depressed \\
Impulsive & Forgiving & Insincere \\
Persistent & Thoughtful & Ungrateful \\
Excitable & Energetic & Forgetful \\
Mannered & Loveable & Shameful \\
Congenial & Outgoing & Deficient \\
Graceful & Valuable & Sluggish \\
\hline
\end{tabular}

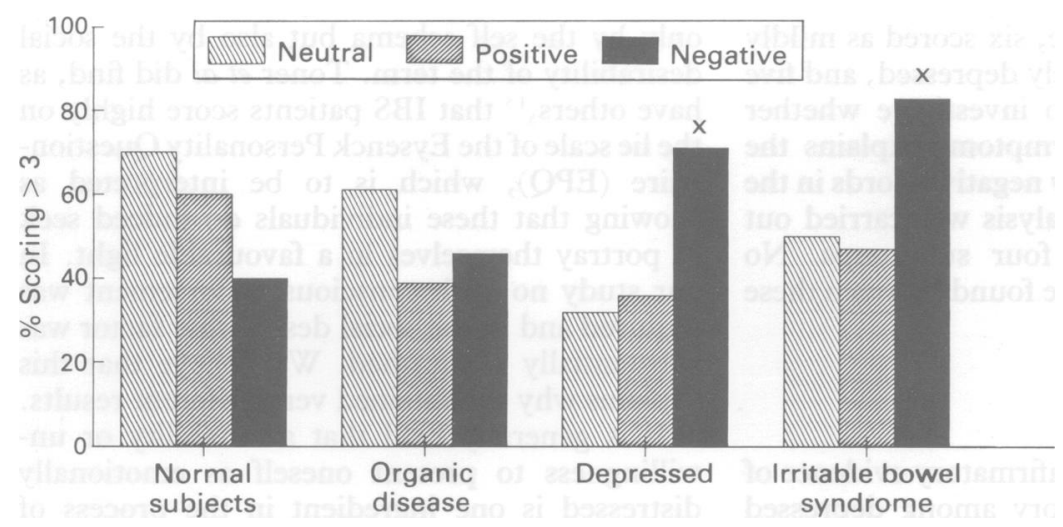

Figure 1: Percentage of subjects from each group with a high correct recognition score $(>3)$ for words in each emotional category.

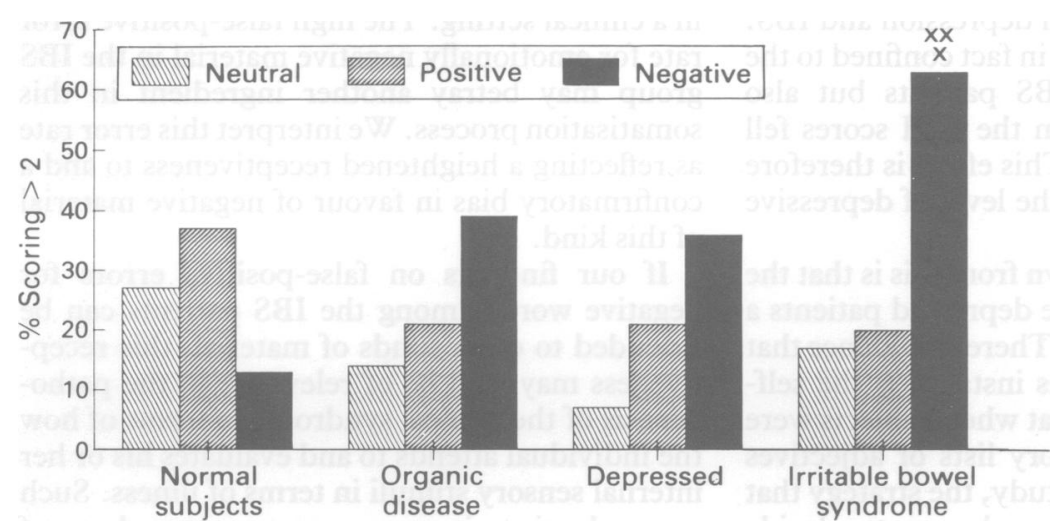

Figure 2: Percentage of subjects in each group with a high false-positive error score $(>2)$ for words in each emotional category.

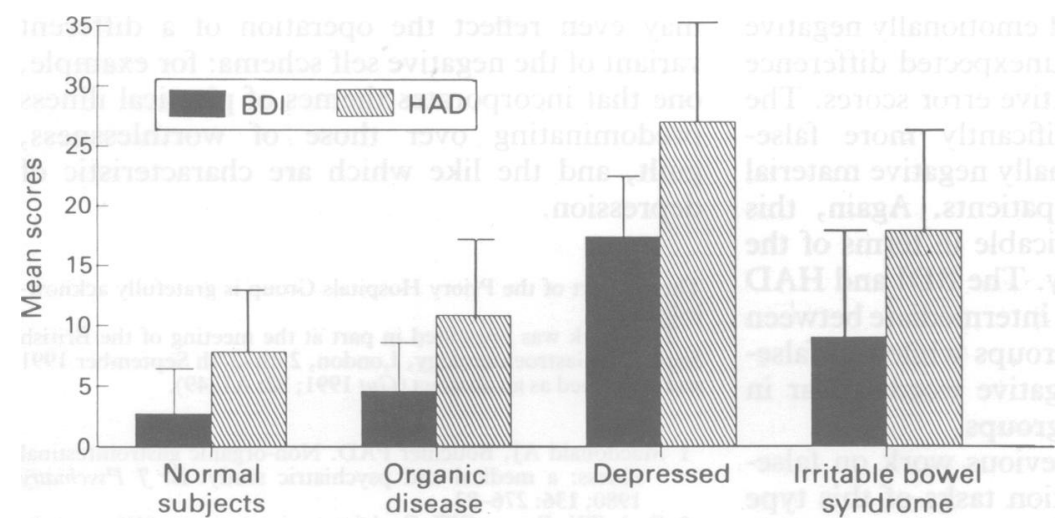

Figure 3: Group mean scores on the Beck Depression Inventory (BDI) and Hospital Anxiety and Depression Scale (HAD). presented with a list of 30 words (10 neutral, 10 positive, 10 negative) and they were asked to tick those words they remembered having been shown previously. In fact only 15 of the words were from the memory task and 15 were new words drawn from the same source and controlled in the same way.

\section{MOOD SCALES}

During the 15 minute interval the subject was asked to complete two self rating mood scales, the Hospital Anxiety and Depression Scale $(\mathrm{HAD})^{10}$ and the short form of the Beck Depression Inventory (BDI)."

\section{STATISTICAL METHODS}

The method we chose was to use $\chi^{2}$ analyses to compare the number of subjects from each group with a high or low correct recognition and false positive error score for words in each emotional category. A high correct recognition score was defined as more than three of five words correctly identified; a high false-positive error score as more than two of five words incorrectly identified. An alternative method of analysis was applied to the actual scores but the results are not shown here as it did not alter the conclusions in any way. HAD and BDI scores were compared using the Wilcoxon rank sum test.

\section{Results}

Figure 1 shows the percentage of subjects from each group with a high correct recognition score (more than 3) for words in each emotional category. The healthy volunteers showed a pattern of recognising more neutral than positive and more positive than negative words. The organic disease group did not differ significantly from this pattern. Both the depressed patients and the IBS patients recognised significantly more emotionally negative words than either the healthy volunteers or the organic disease group $(\mathrm{p}<0.05)$. There was no significant difference in this respect between the depressed patients and the IBS patients.

Figure 2 shows the percentage of subjects from each group with a high false-positive error score (greater than 2 ) for words in each emotional category. The healthy volunteers showed a pattern of incorrectly identifying more positive words than either neutral or negative words. There were no significant differences across the groups for the false-positive error scores for either the emotionally neutral or positive words. For emotionally negative words the picture was different. There was no significant differences between the organic and depressed groups, but both differed significantly from the healthy volunteers $(p<0.05)$. The most striking finding, however, was that the IBS group made significantly more errors for negative words than either the depressed patients $(p<0.05)$, the organic group $(p<0.05)$, or the healthy volunteers $(\mathrm{p}<0.01)$.

Figure 3 shows the group mean scores on the BDI and the HAD anxiety and depression subscales. For the short form of the BDI a score 
of 0-4 rates as normal, 5-7 rates as mild depression, $8-15$ as moderate depression, and over 15 as severe depression. The healthy volunteers (mean (SD) 2.7 (2.6)) and organic disease group $(4 \cdot 6(5 \cdot 2))$ were within the normal range, while the IBS patients $(9 \cdot 1(6 \cdot 5))$ showed moderate depression and the depressed patients (17.4 $(5 \cdot 9))$ showed severe depression. On the HAD, the mean (SD) for the healthy volunteers was $7 \cdot 8$ $(5.0)$, that for the organic disease group was 10.9 (6.9), for the IBS group $18 \cdot 0(7 \cdot 3)$, and for the depressed group $27 \cdot 0(7 \cdot 3)$. On both scales the difference between the scores for the healthy volunteers and organic disease group was not significant. All other differences were highly significant $(\mathrm{p}<0.01)$.

Within the IBS group, six patients had BDI scores in the normal range, six scored as mildly depressed, 13 as moderately depressed, and five as severely depressed. To investigate whether the level of depressive symptoms explains the high scores for emotionally negative words in the IBS group, a separate analysis was carried out on the scores of these four subgroups. No significant differences were found between these subgroups.

\section{Discussion}

In our study we found confirmatory evidence of affective biasing in memory among depressed patients who selectively recognised emotionally negative words. We found the same kind of negative bias among the IBS patients. This is what we had expected to find in view of the known association between depression and IBS. However, this bias was not in fact confined to the depressed subgroup of IBS patients but also occurred in those in whom the BDI scores fell within the normal range. This effect is therefore not simply a reflection of the level of depressive morbidity.

The inference to be drawn from this is that the IBS patients share with the depressed patients a similarly negative schema. There is evidence that the relevant schema in this instance is the selfschema. Rogers ${ }^{12}$ found that when subjects were asked to commit to memory lists of adjectives such as those used in this study, the strategy that they spontaneously employed was to decide covertly while they were memorising whether the item described themselves or not.

Having found this expected similarity between the depressed and the IBS patients in the selective recognition of emotionally negative words, we also found an unexpected difference in terms of their false-positive error scores. The IBS patients made significantly more falsepositive errors for emotionally negative material than did the depressed patients. Again, this finding is not clearly explicable in terms of the level of affective morbidity. The BDI and HAD scores in the IBS group are intermediate between the depressed and other groups while the falsepositive error rate for negative words is far in excess of that in the other groups.

There is a dearth of previous work on falsepositive errors in recognition tasks of this type to assist in the interpretation of this finding. It has been reported, however, that false-positive errors increase as the adjectives become more self descriptive. ${ }^{13}$ The interpretation of our findings in these terms could provide further, albeit indirect, support of our contention that the IBS patients have a negative self schema.

In a recent study Toner et $a l^{1+}$ came to the opposite conclusion - that IBS patients do not have a negative self schema. In their study they found that a group of IBS patients recalled significantly fewer depressed words and more non-depressed words than a group of depressed patients.

However, their procedure was quite different from ours in that it required the explicit endorsement of adjectives as self descriptive or not before an incidental recall task. Such an act of conscious endorsement may be influenced not only by the self schema but also by the social desirability of the term. Toner et al did find, as have others, ${ }^{15}$ that IBS patients score highly on the lie scale of the Eysenck Personality Questionnaire (EPQ), which is to be interpreted as showing that these individuals do indeed seek to portray themselves in a favourable light. In our study no such conscious endorsement was required and so the social desirability factor was substantially diminished. We believe that this explains why we obtained very different results.

It is generally held that an inability or unwillingness to present oneself as emotionally distressed is one ingredient in the process of somatisation, which is the mechanism by which this distress may be manifested as physical symptoms. We believe that such a mechanism may operate in many people with IBS as it is seen in a clinical setting. The high false-positive error rate for emotionally negative material in the IBS group may betray another ingredient in this somatisation process. We interpret this error rate as reflecting a heightened receptiveness to and a confirmatory bias in favour of negative material of this kind.

If our findings on false-positive errors for negative words among the IBS patients can be extended to other kinds of material, this receptiveness may well be of relevance to the pathogenesis of the clinical syndrome in terms of how the individual attends to and evaluates his or her internal sensory stimuli in terms of illness. Such an evaluation is a necessary antecedent of medical consultation and therefore underpins the dimension of healthcare seeking behaviour which is part of the clinical syndrome. ${ }^{16}$

This distinguishing cognitive feature in IBS may even reflect the operation of a different variant of the negative self schema: for example, one that incorporates themes of physical illness predominating over those of worthlessness, guilt, and the like which are characteristic of depression.

The support of the Priory Hospitals Group is gratefully acknowledged.

This work was presented in part at the meeting of the British Society of Gastroenterology, London, 25th-27th September 1991 and published as an abstract (Gut 1991; 32: A1249).

1 Macdonald AJ, Bouchier PAD. Non-organic gastrointestinal illness: a medical and psychiatric study. $\mathrm{Br} \mathcal{F}$ Psychiatry 1980; 136: 276-83.

2 Craig TK, Brown GW. Goal frustrating aspects of life stress in the aetiology of gastrointestinal disorder. $\mathcal{F}$ Psychosom Res 1984; 28: 411-21. 
3 Beck AT. Depression: clinical experimental and theoretical aspects. New York: Hoeber, 1967.

4 Blaney PH. Affect and memory: a review. Psychol Bulletin 1986; 99: $229-46$.

5 Derry PA, Kuiper NA. Schematic processing and self reference in clinical depression. $\mathcal{F}$ Abnorn Psychol 1981; 90: 286-97.

6 Manning AP, Thompson WG, Heaton KW, Morris AF. Towards a positive diagnosis of the irritable bowel syndrome. $B M \mathcal{A}$ 1978; ii: $653-4$.

7 Diagnostic and statistical manual. 3rd ed revised (DSM-III-R). Washington: American Psychiatric Association, 1987.

8 Anderson NH. Likableness ratings of 555 personality trait words. F Pers Soc Psychol 1968; 9: 272-9.

9 Francis WN, Kucera H. Frequence analysis of English usage. Providence: Brown University Press, 1967.

10 Zigmond AS, Snaith RP. The hospital anxiety and depression scale. Acta Psychiat Scand 1983; 67: 361-70.
11 Beck AT, Beck RW. Screening depressed patients in family practice: a rapid technique. Postgrad Med 1972; 52: 81-5.

12 Rogers TB. Self reference in memory: recognition of personality items. F Res Pers 1977; 11: 295-305.

13 Rogers TB, Kuiper NA, Kirker WS. Self-reference and the encoding of personal information. $\mathcal{F}$ Pers Soc Psychol 1977: 35: 677-88.

14 Toner B, Garfinkel P, Jeejeebhoy K, Scher H, Shulhan D. Self-schema in irritable bowel syndrome and depression. Psychosomatic Medicine 1990; 52: 149-55.

15 Latimer PR, Sarna SK, Campbell D, Waterfall W, Daniel E. Colonic motor and myoelectric activity: a comparative study of normal subjects, psychoneurotic patients, and patients with irritable bowel syndrome (IBS). Gastroenterology 1983; 80: 893-901.

16 Sandler RS, Drossman DA, Nathan HP, McKee DC. Symptom complaints and health care seeking behaviour in subjects complaints and health care seeking behaviour in subjects
with bowel dysfunction. Gastroenterology $1984 ; 87: 314-8$. 